\title{
Determinants of postnatal care service utilization among mothers of Mangochi district, Malawi: a community-based cross- sectional study
}

\author{
Jonas Sagawa' ${ }^{1}$ Allen Kabagenyi ${ }^{2}$, Godwin Turyasingura ${ }^{3}$ and Saul Eric Mwale ${ }^{4^{*}}$ (B)
}

\begin{abstract}
Background: Postnatal care (PNC) service is a neglected yet an essential service that can reduce maternal, neonatal and infant morbidity and mortality rates in low and middle-income countries. In Malawi, maternal and infant mortality rates remain high despite numerous efforts by the government and its partners to improve maternal health service coverage across the country. This study examined the determinants of PNC utilization among mothers in Mangochi District, Malawi.

Methods: A community based cross-sectional study was conducted among 600 mothers who gave birth in the past 2 years preceding January 1-31; 2016. A multistage sampling technique was employed to select respondents from nine randomly selected villages in Mangochi district. A transcribed semi-structured questionnaire was pretested, modified and used to collect data on socio-demographic characteristics and maternal related factors. Data was coded in EpiData version 3.1 and analysed in Stata version 12. A multivariable logistic regression adjusted for confounding factors was used to identify predictors of PNC utilization using odds ratio with $95 \%$ confidence interval and $p$-value of 0.05 .

Results: The study revealed that the prevalence of PNC service utilization was $84.8 \%$. Mother's and partner's secondary education level and above $(A O R=2.42, C l: 1.97-6.04 ; A O R=1.45, C l: 1.25-2.49)$, partner's occupation in civil service and business (AOR $=3.17, \mathrm{Cl}$ : 1.25, 8.01; $\mathrm{AOR}=3.39, \mathrm{Cl}: 1.40-8.18$ ), household income of at least MK50, 000 (AOR = 14.41, Cl: 5.90-35.16), joint decision making ( $\mathrm{AOR}=2.27, \mathrm{Cl}: 1.13,4.57)$, knowledge of the available PNC services ( $A O R=4.06, \mathrm{Cl}: 2.22-7.41)$, knowledge of at least one postpartum danger sign ( $A O R=4.00, \mathrm{Cl}: 2.09,7.50$ ), health facility delivery of last pregnancy $(A O R=6.88, C l: 3.35,14.14)$ positively associated with PNC service utilization.
\end{abstract}

* Correspondence: saul.mwale@gmail.com

${ }^{4}$ Biological Sciences Department, Mzuzu University, Private Bag 201, Luwinga, Mzuzu 2, Malawi

Full list of author information is available at the end of the article

(c) The Author(s). 2021 Open Access This article is licensed under a Creative Commons Attribution 4.0 International License, which permits use, sharing, adaptation, distribution and reproduction in any medium or format, as long as you give appropriate credit to the original author(s) and the source, provide a link to the Creative Commons licence, and indicate if changes were made. The images or other third party material in this article are included in the article's Creative Commons licence, unless indicated otherwise in a credit line to the material. If material is not included in the article's Creative Commons licence and your intended use is not permitted by statutory regulation or exceeds the permitted use, you will need to obtain permission directly from the copyright holder. To view a copy of this licence, visit http://creativecommons.org/licenses/by/4.0/. The Creative Commons Public Domain Dedication waiver (http://creativecommons.org/publicdomain/zero/1.0/) applies to the data made available in this article, unless otherwise stated in a credit line to the data. 
Conclusion: The rate of PNC service utilization among mothers was $85 \%$. The uptake of PNC services among mothers was mainly influenced by mother and partner education level, occupation status of the partner, household income, decision making power, knowledge of available PNC services, knowledge of at least one postpartum danger signs, and place of delivery. Therefore, PNC awareness campaigns, training and economic empowerment programs targeting mothers who delivered at home with primary education background and low economic status are needed.

Keywords: Postnatal care, Cross-sectional study, Multistage sampling, Multivariable model, Mangochi district, Malawi

\section{Background}

Postnatal care services, as it is the case with antenatal care, labour and delivery care services, are the fundamental element of the continuum of Essential Obstetric Care (EOC) that can help in decreasing maternal, neonatal, and infant morbidity and mortality in low and middle income countries [1]. According to World Health Organization (WHO), postnatal period begins immediately after the live birth of the baby and extends up to 6 weeks (42 days) after birth and it includes an integrated package of routine maternal and neonatal care [2]. WHO recommends that a mother and her new-born child should receive postnatal care within $24 \mathrm{~h}$ of birth, and then at least three more times, that is to say; on day three, in the second week, and 6 weeks after the birth [2]. Appropriate PNC in the first hours and days following childbirth prevents the great majority of maternal and child morbidity and mortality [3, 4]. It is the period where the health care provider helps mothers and new-borns to establish and maintain contact with a number of health care services needed in the short and long term [3].

Each year, an estimated 529,000 women die from complications related to pregnancy and with $99 \%$ of these maternal deaths occurring in developing countries especially South Asia and sub-Saharan Africa (SSA) [4-6]. In Malawi, like many other SSA countries, maternal and child mortality rates are still high $[7,8]$. Recent estimates showed that the country still experiences maternal and infant mortality rates of 439/100,000 live births and 27/ 1000 live births respectively [8]. Despite its importance, postnatal period is generally the most neglected in developing countries [9] including Malawi. At national level, PNC utilization within 2 weeks of delivery is currently at 42\% [8]. In Mangochi district PNC utilization within 2 weeks of delivery is currently at $28 \%$ [10]. Understanding the factors related to PNC utilization is critical for countries like Malawi with an alarmingly high maternal, neonatal and infant mortality levels. Recently, studies on determinants of postnatal care utilization have been conducted in Sub-Saharan Africa, Malawi inclusive [11-26]. These studies have provided insights into the many multilevel factors that influence postnatal care utilization. Since, the determinants of PNC utilization are not the same across different cultures, different geographical locations and socio-economic status within a society [19]. This study therefore was conceptualized to examine the determinants of postnatal care service utilization among mothers in rural communities of Mangochi district, Malawi.

\section{Methods \\ Study setting, design and sampling technique}

A community based cross-sectional study was carried out from 1st to 31st January, 2016 in Mangochi district which is located in the eastern region of Malawi. The study participants were drawn from nine randomly selected villages in the district from three Traditional $\mathrm{Au}-$ thorities; Chilipa, Makanjira, and Nankumba. Six hundred women were selected using a multistage sampling technique.

\section{Study population and sampling procedure}

Mothers who gave birth in the past 2 years preceding data collection period, with a child more than 6 weeks old, willing to participate and living in Mangochi district were included. Multistage sampling technique was used to select study subjects. Initially, all the 12 Traditional Authorities in the district were stratified into three, with four neighbouring Traditional Authorities being put in one stratum based on their geographical location. Then one Traditional Authority was selected randomly from each stratum to come up with the three Traditional Authorities to be representatives for the study. From the chosen Traditional Authorities, frame of the villages in each was drawn and three villages were chosen from each of the chosen Traditional Authorities using systematic random sampling technique. Sample size was allocated proportionally to each randomly selected village. Finally, frames of households with eligible woman were prepared for each village. Whenever more than one eligible respondent was found in the same selected household, only one respondent was chosen by lottery method.

Sample size was determined using Leslie Kish [27] sampling formula by considering $28 \%$ PNC utilization rate in Mangochi district [10], 95\% level of confidence 
and 5\% margin of error. A design effect of 1.5 and $20 \%$ non-response rate were also anticipated to obtain the final sample size of approximately 600 .

\section{Data collection tools and procedure}

The structured questionnaire was prepared using concepts in the conceptual framework and study objectives and utilized in data collection. The questionnaire was prepared in English (See Supplementary File 1) and then translated in Chichewa which is the common local language used by almost everyone in the district (See Supplementary File 2). To check its consistency, the questionnaire was back translated to English by an expert of both languages. The questionnaire was pre-tested on $10 \%$ of the population outside the study area, modified and used for data collection. Five research assistants who were fluent in the local language (Chichewa) and familiar with the local customs were recruited and used to collect the data. One research assistant who had vast experience in household surveys was assigned to supervise the data collection process. A day training was given on the objective of the study, confidentiality of information and the techniques of an interview to data collectors and supervisors by the Principal Investigator.

\section{Data analysis}

The collected data was entered and coded in EpiData version 3.1 and analysed for both descriptive and inferential statistics using Stata Version 12 software [28]. Data analysis involved univariate, bivariate and multivariate analysis. Descriptive statistics involved generating summary statistics and frequency distribution for sociodemographic characteristics of the respondents and maternal related factors. Inferential statistics through a Pearson chi-square test was used to measure the association of socio-demographic characteristics and maternal related factors with postnatal care service utilization. A multivariable logistic regression was performed on variables such as mother's education level, partner's education level, occupation of the partner, age of respondents, marital status, parity, household income, decision making, knowledge of PNC services, knowledge of the postpartum danger signs and place of delivery based on either their significance in predicting PNC utilization from literature or that they were significantly associated with postnatal care service utilization at a bivariate level of analysis $(p<0.05)[12,17]$ in order to identify the predictors of postnatal care service utilization. A multicollinearity test for individual level predictors was performed in order to control the inflationary effect of standard errors due to many predictors measuring the same variable. The variance inflation factor (VIF) and tolerance for this study were within the acceptable levels of less than or equal to 2.5 and greater than or equal to 0.4 respectively (See Supplementary file 4) for logistic regression [29]. Knowledge of PNC services, Knowledge of Postnatal danger signs and Place of delivery predictors were confounding factors since the difference in their regression coefficients between unadjusted and adjusted models was greater than 20\% [30]. However, the confounding factors were maintained in the model. The model sufficiently described the data with a smaller value of Akaike's Information Criterion (AIC) (See Supplementary File 5) and non-significant Hosmer Lemeshow test. The Odds Ratios (AOR) associated with these factors were reported as a measure of strength, together with their respective 95\% Confidence Intervals and $p$ value of 0.05 .

\section{Results}

\section{Socio-demographic characteristics}

Age, marital status, mother's and partner's education level, occupation status of the mother and partner, household income, distance to health facility, decision making power and family size were assessed as sociodemographic characteristics of the mothers in this study. A total of 600 women of reproductive age (15-49 years) who gave birth in the last 2 years prior to the survey were interviewed making a response rate of $100 \%$. Mothers aged between 20 and 24 years old contributed $38.7 \%$ of the total respondents, followed by those aged 25-29 (21.8\%) and 30-34 (16.0\%), while the rest were either above 34 years $(9.2 \%)$ or below 20 years (Table 1 ). With regards to marital status, slightly over eight out of 10 were in marital union, while the rest were either never married (11.7\%), or divorced/widowed (4.6\%) (Table 1). The majority of the mothers $64.8 \%$ had at least primary education while only $20.7 \%$ had no education at all and the same trend was seen in partners where almost $50 \%$ had secondary education (Table 1). Regarding occupation status, at least half of the mothers $51.2 \%$ were farmers, and $20.6 \%$ were doing business, and for the partners, the majority $42.7 \%$ were farmers, $23.2 \%$ were businessmen, while $19.5 \%$ had no job at all (Table 1 ). About $36 \%$ of the respondents were from the poorest wealth quantile earning less than 20,000 Malawi Kwacha in a month. About $41.5 \%$ of the mothers were residing over $10 \mathrm{~km}$ from the health facility. Slightly above half (50.9\%) of the mothers reported being involved in the decision making regarding postnatal care service utilization. Among these mothers, $27.2 \%$ were able to make the decision by themselves, while $23.7 \%$ reported of making the decision together with their husbands (Table 1). Furthermore, majority $47.0 \%$ of the respondents were from small families, with 2-4 family members, $32.2 \%$ were from medium size family and the remaining proportion were from big family size with at least six family member. 
Table 1 Distribution of respondents by socio-demographic characteristics

\begin{tabular}{|c|c|c|}
\hline Variables & Frequency $(n=600)$ & Percentage (\%) \\
\hline \multicolumn{3}{|l|}{ Age } \\
\hline $15-19$ & 86 & 14.3 \\
\hline $20-24$ & 232 & 38.7 \\
\hline $25-29$ & 131 & 21.8 \\
\hline $30-34$ & 96 & 16.0 \\
\hline $35+$ & 55 & 9.2 \\
\hline \multicolumn{3}{|l|}{ Marital status } \\
\hline Single & 70 & 11.7 \\
\hline Married & 502 & 83.7 \\
\hline Divorced/Widowed & 28 & 4.6 \\
\hline \multicolumn{3}{|c|}{ Respondent's education level } \\
\hline None & 124 & 20.7 \\
\hline Primary & 389 & 64.8 \\
\hline Secondary \& above & 87 & 14.5 \\
\hline \multicolumn{3}{|c|}{ Partner's education level } \\
\hline None & 85 & 15.5 \\
\hline Primary & 192 & 35.0 \\
\hline Secondary \& above & 272 & 49.5 \\
\hline \multicolumn{3}{|c|}{ Occupation of respondent } \\
\hline None & 121 & 20.1 \\
\hline House wife & 48 & 8.0 \\
\hline Business & 124 & 20.7 \\
\hline Farmer & 307 & 51.2 \\
\hline \multicolumn{3}{|l|}{ Occupation of partner } \\
\hline None & 107 & 19.5 \\
\hline Farmer & 234 & 42.7 \\
\hline Business & 127 & 23.2 \\
\hline Civil servant & 80 & 14.6 \\
\hline \multicolumn{3}{|l|}{ Household Income } \\
\hline$<\mathrm{K} 20,000$ & 213 & 35.5 \\
\hline $\mathrm{K} 20,000-\mathrm{K} 50,000$ & 113 & 18.8 \\
\hline K50,000-K150,000 & 208 & 34.7 \\
\hline$>\mathrm{K} 150,000$ & 66 & 11 \\
\hline \multicolumn{3}{|c|}{ Distance to health facility } \\
\hline 0-2 Kilometers & 164 & 27.3 \\
\hline 3-5 Kilometers & 125 & 20.8 \\
\hline 6-10 Kilometers & 62 & 10.4 \\
\hline > 10 Kilometers & 249 & 41.5 \\
\hline \multicolumn{3}{|l|}{ Decision making } \\
\hline Self & 163 & 27.2 \\
\hline Husband & 193 & 32.1 \\
\hline Joint & 142 & 23.7 \\
\hline Other & 102 & 17 \\
\hline
\end{tabular}


Table 1 Distribution of respondents by socio-demographic characteristics (Continued)

\begin{tabular}{|c|c|c|}
\hline Variables & Frequency $(n=600)$ & Percentage (\%) \\
\hline \multicolumn{3}{|l|}{ Family size } \\
\hline 2-4 (Small) & 282 & 47 \\
\hline 5-6 (Medium) & 193 & 32.2 \\
\hline$>6$ (Big) & 125 & 20.8 \\
\hline
\end{tabular}

\section{Maternal related factors}

Regarding the maternal related factors, the following characteristics, including parity, knowledge of postnatal care services, postnatal danger signs and place of delivery were assessed. About $41.5 \%$ of the mothers had 2-3 children and only $9.7 \%$ had more than five children by the time this study was being conducted (Table 2). Mothers were asked whether they have ever heard of available postnatal health care services, and the postnatal danger signs. $84.8 \%$ of the mothers reported having ever heard of available postnatal care services, while the rest $15.2 \%$ said they had never heard of the PNC services (Table 2). Regarding knowledge of postpartum danger signs, at least 8 out of 10 mothers reported to have ever heard of postpartum danger signs. Lastly, the highest proportion of the mothers (89.3\%) delivered at the health facility while the remaining proportion had home deliveries (Table 2).

\section{Postnatal care service utilization}

Postnatal care service utilization was assessed based on binary outcome, yes or no response. In this study, a mother was considered to have utilized postnatal care services if she had attended at least one postnatal care visit within the 6 weeks post-delivery. The majority of the respondents $84.8 \%$ utilized postnatal care services

Table 2 Distribution of respondents by maternal related factors

\begin{tabular}{lcl}
\hline Variables & Frequency $(\boldsymbol{n}=\mathbf{6 0 0})$ & Percentage (\%) \\
\hline Parity & 168 & 28.0 \\
One & 249 & 41.5 \\
$2-3$ & 125 & 20.8 \\
$4-5$ & 58 & 9.7 \\
$>5$ & 509 & \\
Ever heard of PNC services & 84.8 \\
Yes & 91 & 15.2 \\
No & 515 & \\
Heard of postnatal danger signs & 85.8 \\
Yes & 85 & 14.2 \\
No & & \\
Place of delivery & 64 & 10.7 \\
Home & 536 & 89.3 \\
Health facility &
\end{tabular}

while the remaining $15.2 \%$ did not utilize postnatal care services at all (Fig. 1).

\section{Bivariate analysis results of postnatal care service utilization}

The association of postnatal care service utilization with socio-demographic characteristics and maternal related factors results are presented in Tables 3 and 4 respectively. The results show that mother's education level, partner's education level, occupation of the mother and partner, household income, decision making as sociodemographic characteristics were significantly associated with postnatal care service utilization (Table 3). While the following maternal related factors; knowledge of available PNC services, knowledge of postpartum danger signs and place of delivery were significantly associated with PNC service utilization (Table 4).

\section{Socio-demographic predictors of postnatal care service utilization}

Some variables from a bivariate analysis which were not significantly associated with PNC utilization such as mother's age, marital status and parity but were supported by previous relevant literature were considered for inclusion in the logistic regression model. After performing a multicollinearity test as a requirement for regression analysis these variables displayed weak correlations and were included in the regression model. However, at a regression analysis level these variables still were not significantly associated with PNC service utilization (See Supplementary File 3).

The level of education of the mother and that of the partners showed a strong statistical association with postnatal care service utilization (Table 5). Mothers and their partners who attended secondary school were at least 2 times $(\mathrm{AOR}=2.42, \mathrm{CI}: 1.97,6.04)$ and almost 1.5 times $(\mathrm{AOR}=1.45, \mathrm{CI}: 1.25,2.49)$ respectively more likely to utilize postnatal care services than mothers and their partners who had no education at all. Similarly, respondents whose partners were in civil service were at least 3 times $(\mathrm{AOR}=3.17, \mathrm{CI}: 1.25,8.01)$ more likely to utilize postnatal care services than mothers whose partners were not working at all (Table 5). Furthermore, mothers who were from middle income families were at least 14 times $(\mathrm{AOR}=14.41, \mathrm{CI}: 5.90,35.16)$ more likely to utilize postnatal care services than mothers from 


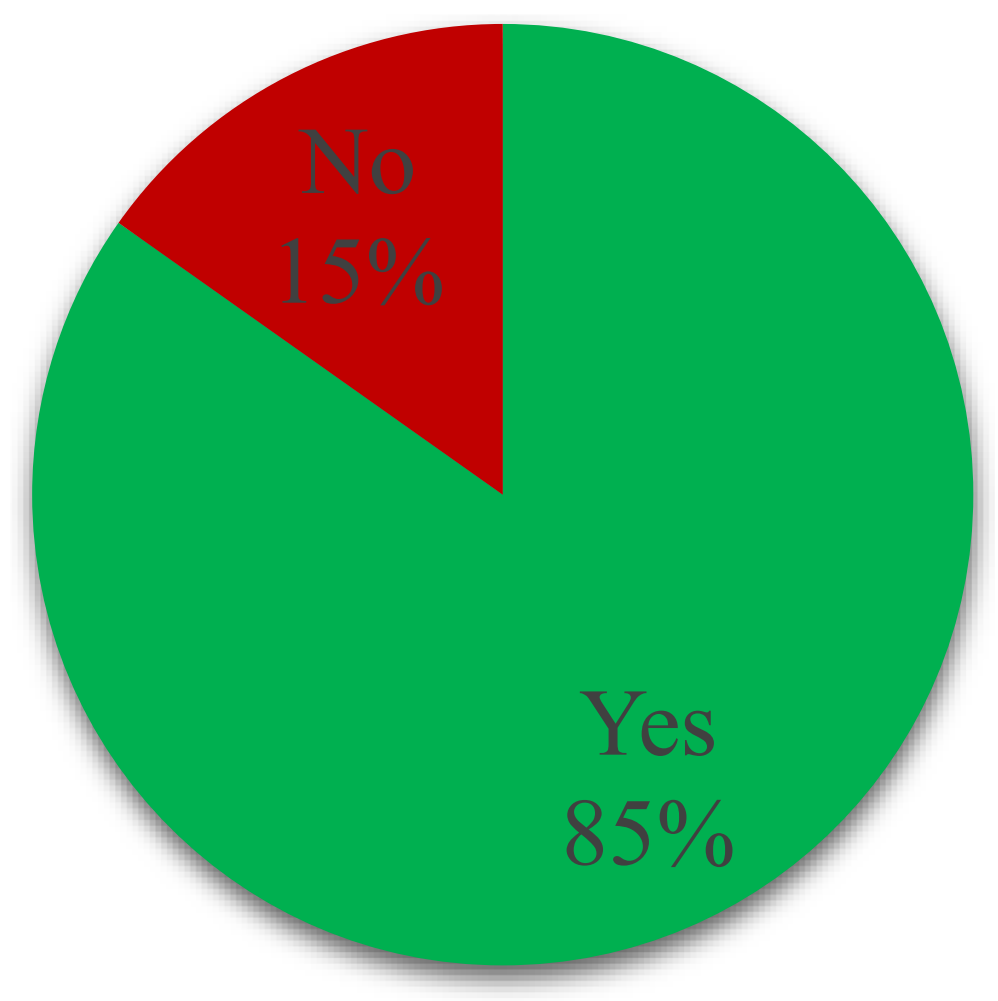

Fig. 1 Distribution of respondents by postnatal care service utilization

poorest families. Lastly, involving the mother in making decision regarding postnatal care service utilization increased at least twice ( $\mathrm{AOR}=2.27, \mathrm{CI}: 1.13,4.57)$ the odds of utilizing postnatal care services among mothers of Mangochi district.

\section{Maternal related predictors of postnatal care service utilization}

As for maternal related factors, place of delivery showed a strong prediction for postnatal care service utilization. Mothers who gave birth with their current last born at the health facility were almost 7 times $(\mathrm{AOR}=6.88, \mathrm{CI}$ : $3.35,14.14)$ more likely to utilize postnatal care services as compared to mothers who delivered at home (Table 6). Probability of postnatal care service utilization also has shown to be highly predicted by awareness of available postnatal care services. In this study, mothers who were aware of the available postnatal care services, when and where to get them were at least 4 times $(\mathrm{AOR}=4.06$, CI: $2.22,7.41)$ more likely to have utilized postnatal care services than mothers who were not aware of available postnatal care services. The study also found that mothers who had knowledge of at least one postnatal obstetric danger sign were 4 times $(\mathrm{AOR}=$ 4.00, CI: $2.09,7.50$ ) more likely to utilize postnatal care services than those mothers who had never heard of postnatal danger signs at all (Table 6).

\section{Discussion}

The study examined the determinants of PNC service utilization among mothers in rural communities of Mangochi district, Malawi. The study revealed that $84.8 \%$ mothers utilized PNC services. The results are slightly higher than those reported by $[11,12,20]$. This may be attributed to the differences in study settings, samples size, study period and other related factors that might have led to the improvement in accessing and utilizing PNC services through time. Despite the higher percentage of PNC utilization by the mothers in the district, it's only $5.5 \%$ of the respondents that met the set criteria of number of PNC visits by $\mathrm{WHO}$ and $\mathrm{MoH}$, Malawi. This clearly indicates that the PNC service in the district is poorly utilized.

Socio-demographic factors such as educational level of the mothers and partners, occupation status of the partner, household level of income determined PNC service utilization. However, PNC service utilization by the mothers varied with the level of education attained by the mothers and their partners. The likelihood of utilizing PNC service was more pronounced among those with secondary level education and above than those with primary level education. The results coincide with the findings of [11-20, 31-33]. This can be attributed to the fact that education serves as a proxy for information, cognitive skills and values which in-turn promotes 
Table 3 Chi-square analysis of socio-demographic characteristics with PNC of the respondents in Mangochi, Malawi $(n=600)$

\begin{tabular}{|c|c|c|c|c|}
\hline \multirow{2}{*}{$\begin{array}{l}\text { Variables } \\
\text { Age }\end{array}$} & \multicolumn{2}{|c|}{ PNC Service Utilization (\%) } & \multirow{2}{*}{$\frac{\text { Chi-statistic (x } \mathbf{2})}{3.03}$} & \multirow{2}{*}{$\frac{\boldsymbol{P} \text {-values }}{0.55}$} \\
\hline & Yes & No & & \\
\hline $15-19$ & 83.7 & 16.3 & & \\
\hline $20-24$ & 84.0 & 16.0 & & \\
\hline $25-29$ & 85.0 & 15.0 & & \\
\hline $30-34$ & 83.3 & 16.7 & & \\
\hline $35+$ & 92.7 & 7.3 & & \\
\hline \multicolumn{5}{|l|}{ Marital status } \\
\hline Single & 81.4 & 18.6 & 1.47 & 0.63 \\
\hline Married & 85.5 & 14.5 & & \\
\hline Divorced/Widowed & 82.1 & 17.9 & & \\
\hline \multicolumn{5}{|c|}{ Respondent's education level } \\
\hline None & 75.8 & 24.2 & 11.78 & 0.00 \\
\hline Primary & 86.1 & 13.9 & & \\
\hline Secondary \& above & 92.0 & 8.0 & & \\
\hline \multicolumn{5}{|c|}{ Partner's education level } \\
\hline None & 75.3 & 24.7 & 8.48 & 0.01 \\
\hline Primary & 86.5 & 13.5 & & \\
\hline Secondary \& above & 87.9 & 12.1 & & \\
\hline \multicolumn{5}{|c|}{ Occupation of respondent } \\
\hline None & 92.6 & 7.4 & 8.65 & 0.03 \\
\hline House wife & 77.1 & 22.9 & & \\
\hline Business & 82.3 & 17.7 & & \\
\hline Farmer & 84.0 & 16.0 & & \\
\hline \multicolumn{5}{|l|}{ Occupation of partner } \\
\hline None & 78.5 & 21.5 & 10.67 & 0.01 \\
\hline Farmer & 83.3 & 16.7 & & \\
\hline Business & 91.3 & 8.7 & & \\
\hline Civil servant & 91.2 & 8.8 & & \\
\hline Household Income & Yes & No & & \\
\hline$<\mathrm{K} 20,000$ & 70.4 & 29.6 & 68.49 & 0.00 \\
\hline $\mathrm{K} 20,000-\mathrm{K} 50,000$ & 88.5 & 11.5 & & \\
\hline K50,000-K150,000 & 96.6 & 3.4 & & \\
\hline$>\mathrm{K} 150,000$ & 94.4 & 5.6 & & \\
\hline \multicolumn{5}{|c|}{ Distance to Health facility } \\
\hline 0-2 Kilometers & 87.2 & 12.8 & 3.44 & 0.33 \\
\hline 3-5 Kilometers & 84.0 & 16.0 & & \\
\hline 6-10 Kilometers & 90.3 & 9.7 & & \\
\hline$>10$ Kilometers & 82.3 & 17.7 & & \\
\hline \multicolumn{5}{|l|}{ Decision making } \\
\hline Self & 77.9 & 22.1 & 9.21 & 0.03 \\
\hline Husband & 87.1 & 12.9 & & \\
\hline Joint & 85.9 & 14.1 & & \\
\hline Other & 90.2 & 9.8 & & \\
\hline
\end{tabular}


Table 3 Chi-square analysis of socio-demographic characteristics with PNC of the respondents in Mangochi, Malawi $(n=600)$ (Continued)

\begin{tabular}{llll}
\hline Variables & PNC Service Utilization (\%) & Chi-statistic (X2) & $P$-values \\
\hline Family size & & & 1.94 \\
2-4 (Small) & 83.7 & 16.3 & \\
5-6 (Medium) & 83.9 & 16.1 & \\
$>$ 6 (Big) & 88.8 & 11.2 & \\
\hline
\end{tabular}

health seeking behavior through increased level of awareness of the available health services $[11,19]$. Family disposable income obtained through an occupation of the partner or an income generating activity and joint decision making influenced PNC service utilization. Those with the ability to pay for the health care service for their wives were more likely to utilize PNC service than those without the ability to pay for the PNC service. Mothers who were involved in making decision regarding postnatal care service utilization were more likely to have utilized PNC services more than those who had to wait for either their husbands/partners or other significant others to make the decision for them [12, 21, 34-36]. reported similar findings.

Knowledge of the available PNC service, postpartum obstetric danger signs and place of delivery associated with PNC service utilization. Mothers who were aware of the available PNC service and postpartum obstetric danger signs were more likely to utilize PNC service than those who were not aware. Previous studies in developing countries also found that knowledge of postpartum danger signs and knowledge of available PNC service influences the uptake of PNC services [9, 31, 34]. This can be explained by the fact that awareness of obstetric danger sign is an important factor in triggering and motivating the mothers to seeking health care services with the aim of preventing, diagnosing and even treating the possible postnatal danger sign [19]. Additionally, knowledge is an important factor in the utilization of PNC services as it enables one to understand the services that are available, their importance, when and where to get them [21].

Mothers who delivered their last baby in the health facility utilized PNC services more when compared with those who delivered at home. This finding aligns well with the results from a study conducted in Madhya Pradesh, India [32] that reported that women who delivered at health facility utilized PNC services more than those who delivered elsewhere. This can be attributed to the fact that women who gave birth in health institutions have greater chances to get exposed to health education related to PNC services at the time of delivery and thus get access to learn about the types, benefits, and availabilities of PNC services, where and when to access them, during their stay in the health institution [19].

\section{Limitations of the study}

Recall biasness by respondents is one of the limitation of the study as only mothers who had given birth in the past 2 years preceding data collection period were

Table 4 Chi-square analysis of maternal related factors with PNC of the respondents in Mangochi, Malawi $(n=600)$

\begin{tabular}{|c|c|c|c|c|}
\hline \multirow{2}{*}{$\frac{\text { Variables }}{\text { Parity }}$} & \multicolumn{2}{|c|}{ Postnatal Service Utilization (\%) } & \multirow[t]{2}{*}{ Chi-statistic ( $\left.x^{2}\right)$} & \multirow[t]{2}{*}{$P$-values } \\
\hline & Yes & No & & \\
\hline One & 84.5 & 15.5 & 1.72 & 0.63 \\
\hline $2-3$ & 85.5 & 14.5 & & \\
\hline $4-5$ & 86.4 & 13.6 & & \\
\hline$>5$ & 79.3 & 20.7 & & \\
\hline \multicolumn{5}{|c|}{ Ever heard of PNC services } \\
\hline Yes & 89.0 & 11.0 & 45.24 & 0.00 \\
\hline No & 61.5 & 38.5 & & \\
\hline \multicolumn{5}{|c|}{ Heard of postpartum danger signs } \\
\hline Yes & 89.3 & 10.7 & 56.89 & 0.00 \\
\hline No & 57.7 & 42.3 & & \\
\hline \multicolumn{5}{|l|}{ Place of delivery } \\
\hline Home & 46.9 & 53.1 & 80.23 & 0.00 \\
\hline Health facility & 89.4 & 10.6 & & \\
\hline
\end{tabular}


Table 5 Logistic regression analysis of Socio-demographic characteristics associated with PNC utilization of mothers in Mangochi, Malawi $(n=600)$

\begin{tabular}{|c|c|c|c|c|c|}
\hline \multirow{2}{*}{$\begin{array}{l}\text { Variables } \\
\text { Mother's education level }\end{array}$} & \multicolumn{2}{|c|}{ PNC Utilization (\%) } & \multirow[t]{2}{*}{ COR (Cl) } & \multirow[t]{2}{*}{ AOR (Cl) } & \multirow[t]{2}{*}{$P$-values } \\
\hline & Yes & No & & & \\
\hline None $^{\mathrm{R}}$ & 75.8 & 24.2 & 1.00 & 1.00 & 1.00 \\
\hline Primary & 86.1 & 13.9 & $1.98(1.19-3.28)$ & $1.80^{* *}(1.01-3.19)$ & 0.005 \\
\hline Secondary \& above & 92.0 & 8.0 & $3.65(1.49-8.94)$ & $2.31^{* *}(1.97-6.04)$ & 0.001 \\
\hline \multicolumn{6}{|l|}{ Partner's education level } \\
\hline None ${ }^{R}$ & 75.3 & 24.7 & 1.00 & 1.00 & 1.00 \\
\hline Primary & 86.5 & 13.5 & $2.09(1.09-4.02)$ & $1.66(0.88-3.13)$ & 0.115 \\
\hline Secondary \& above & 87.9 & 12.1 & $2.38(1.28-4.42)$ & $1.45^{* *}(1.25-2.49)$ & 0.004 \\
\hline \multicolumn{6}{|l|}{ Occupation of partner } \\
\hline None $^{R}$ & 78.5 & 21.5 & 1.00 & 1.00 & 1.00 \\
\hline Farmer & 83.3 & 16.7 & $1.37(0.77-2.44)$ & $2.38^{*}(1.20-4.72)$ & 0.039 \\
\hline Business & 91.3 & 8.7 & $2.89(1.32-6.34)$ & $3.39^{* *}(1.40-8.18)$ & 0.006 \\
\hline Civil servant & 91.2 & 8.8 & $2.86(1.14-7.15)$ & $3.17^{*}(1.25-8.01)$ & 0.014 \\
\hline \multicolumn{6}{|l|}{ Household Income } \\
\hline$<K 20,000^{R}$ & 70.4 & 29.6 & 1.00 & 1.00 & 1.00 \\
\hline$K 20,000-K 50,000$ & 88.5 & 11.5 & $3.23(1.66-6.27)$ & $2.82^{* * *}(1.40-5.70)$ & 0.000 \\
\hline K50,000-K150,000 & 96.6 & 3.4 & $12.06(5.08-28.65)$ & $14.41^{* * *}(5.90-35.16)$ & 0.000 \\
\hline$>$ K150,000 & 94.4 & 5.6 & 7.14 (2.08-24.54) & $4.63^{* * *}(1.43-15.03)$ & 0.000 \\
\hline \multicolumn{6}{|l|}{ Decision making } \\
\hline Self ${ }^{R}$ & 77.9 & 22.1 & 1.00 & 1.00 & 1.00 \\
\hline Husband & 87.1 & 12.9 & $1.90(1.08-3.35)$ & $1.60^{*}(1.48-2.89)$ & 0.017 \\
\hline Joint & 85.9 & 14.1 & $1.73(0.94-3.17)$ & $2.27^{*}(1.13-4.57)$ & 0.031 \\
\hline Other & 90.2 & 9.8 & $2.61(1.22-5.58)$ & $1.96^{* *}(1.85-4.50)$ & 0.007 \\
\hline
\end{tabular}

${ }^{*} p<0.05,{ }^{* *} p<0.01,{ }^{* * *} p<0.001$, Note $\mathrm{R}=$ Reference category

considered. Cause and effect of the predictors could not be established due to the type of research design used.

\section{Conclusions}

PNC services are a significant constituent of safe motherhood. The study revealed that the overall utilization of PNC services among mothers in Mangochi district, Malawi was good as almost $85 \%$ of the mothers were able to utilize the services, while $15 \%$ of them had poor utilization of postnatal care services. Among the PNC service utilizers, only 5.5\% utilized PNC services more than three times as recommended by $\mathrm{WHO}$ and

Table 6 Logistic regression analysis for the association between maternal-related factors and postnatal care utilization in Mangochi, Malawi $(n=600)$

\begin{tabular}{|c|c|c|c|c|c|}
\hline \multirow{2}{*}{$\begin{array}{l}\text { Variables } \\
\text { Ever heard of PNC services }\end{array}$} & \multicolumn{2}{|c|}{ PNC Utilization (\%) } & \multirow[t]{2}{*}{$\operatorname{COR}(\mathrm{CI})$} & \multirow[t]{2}{*}{ AOR (Cl) } & \multirow[t]{2}{*}{$P$-values } \\
\hline & Yes & No & & & \\
\hline $\mathrm{No}^{\mathrm{R}}$ & 61.5 & 38.5 & 1.00 & 1.00 & 1.00 \\
\hline Yes & 89.0 & 11 & $5.06(3.00-8.56)$ & $4.06^{* * *}(2.22-7.41)$ & 0.000 \\
\hline \multicolumn{6}{|c|}{ Heard of postnatal danger signs } \\
\hline $\mathrm{No}^{\mathrm{R}}$ & 57.7 & 42.3 & 1.00 & 1.00 & 1.00 \\
\hline Yes & 89.3 & 10.7 & $6.14(3.58-10.54)$ & $4.00^{* * *}(2.09-7.50)$ & 0.000 \\
\hline \multicolumn{6}{|l|}{ Place of delivery } \\
\hline Home $^{R}$ & 46.9 & 53.1 & 1.00 & 1.00 & 1.00 \\
\hline Health facility & 89.4 & 10.6 & $9.52(5.20-17.44)$ & $6.88^{* * *}(3.35-14.14)$ & 0.000 \\
\hline
\end{tabular}

***p $<0.001$. Note R= Reference category 
Ministry of Health, Malawi. The study also established determinants of PNC utilization which should be targeted through health care awareness and sensitization campaigns on the available PNC services to enhance uptake of PNC services among mothers.

\author{
Abbreviations \\ AOR: Adjusted Odds Ratio; COR: Crude Odds Ratio; Cl: Confidence Interval; \\ EOC: Essential Obstetric Care; IEC: Information Education and \\ Communication; PNC: Postnatal Care; USAID: United States Agency for \\ International Development; WHO: World Health Organization: \\ Coef.: Coefficient; MOH: Ministry of Health
}

\section{Supplementary Information}

The online version contains supplementary material available at https://doi. org/10.1186/s12884-021-04061-4.

Additional file 1: Supplementary File 1. Questionnaire in English. Additional file 2: Supplementary File 2. Questionnaire Translated from English to Chichewa.

Additional file 3: Supplementary File 3. Logistic regression analysis for postnatal care service utilization on socio-demographic and maternal related factors.

Additional file 4: Supplementary File 4. Results of multicollinearity test for selected predictors for the multivariable model.

Additional file 5: Supplementary File 5. Regression diagnostics.

\section{Acknowledgments}

We thank the United States Agency for International Development (USAID) through World Learning Malawi Scholarship program for providing financial support towards the study. The authors would also like to thank National Commission for Science and Technology (NCST), Malawi, and Mangochi District Health Office for the permission and support they provided towards the accomplishment of the study. We are also grateful to all the village heads of all the villages that participated in this study for the permission and support given to us during the time the data was collected in their respective villages. Lastly, our great appreciation goes to the respondents for sacrificing their time in providing data for this study. The contents are solely the responsibility of the authors and do not necessarily represent the official views of the supporting offices.

\section{Authors' contributions}

JS, AK and GT contributed to the concept of the study. JS and SEM performed statistical analysis. JS, AK and SEM jointly wrote the draft manuscript. JS, AK, GT and SEM contributed in interpreting the result, literature review and revising the manuscript. All authors revised the manuscript and agreed on the findings and views expressed. All authors read, approved the final version of the manuscript and agreed to abide by the copyright terms and conditions of BioMed Central.

\section{Funding}

This research was financially supported by United States Agency for International Development (USAID) through World Learning Malawi Scholarship program. The funder did not take part in the design of the study, data collection, data analysis and interpretation and in writing the manuscript.

\section{Availability of data and materials}

The data sets used and analysed during the current study will be made available upon reasonable request made through the corresponding author.

\section{Declarations}

Ethics approval and consent to participate

Ethical clearance was obtained from the Higher Degree Ethics and Research Committee of Makerere University School of Statistics and Planning, and the National Commission for Science and Technology (NCST) of Malawi Protocol
Number P.12/15/67. Permission to conduct the study was obtained from Mangochi District Health Office. A written informed consent was obtained from the village head of each selected village of study, and from each respondent. Respondents were informed that all the information obtained from them would be kept confidential. Furthermore, the participants were informed of their right to withdrawal from the study anytime they felt like with no consequences attached. The questionnaire was coded instead of using names as identification and hence confidentiality was assured throughout the study.

\section{Consent for publication}

Not applicable.

\section{Competing interests}

The authors declare that there is no conflict of interest.

\section{Author details}

${ }^{1}$ Community Health Department, Kamuzu University of Health Sciences, Private Bag 360, Chichiri, Blantyre 3, Malawi. ${ }^{2}$ School of Statistics and Planning, Department of Population Studies, Makerere University, Post Office Box 7062, Kampala, Uganda. ${ }^{3}$ Kabale University, Post office Box 317, Kabale, Uganda. ${ }^{4}$ Biological Sciences Department, Mzuzu University, Private Bag 201, Luwinga, Mzuzu 2, Malawi.

Received: 28 October 2020 Accepted: 18 August 2021

Published online: 30 August 2021

\section{References}

1. Say L, Raine R. A systematic review of inequalities in the use of maternal health care in developing countries: examining the scale of the problem and the importance of context. Bull World Health Organ. 2007;85(10):812-9. https://doi.org/10.2471/blt.06.035659. PMID: 18038064; PMCID: PMC2636485.

2. WHO. WHO Technical consultation on postpartum and postnatal care report. Geneva: World Health Organization; 2010. p. 1-56.

3. WHO, UNICEF, UNFPA, The World Bank. Trends in maternal mortality:1990 to 2013. Geneva: World Health Organization; 2014.

4. Nour NM. An introduction to maternal mortality. Rev Obstet Gynecol. 2008; 1(2):77-81. Spring; PMID: 18769668; PMCID: PMC2505173.

5. Sines E, Syed U, Wall S, Worley H. Postnatal care: a critical opportunity to save mothers and newborns. Washington DC: Population Reference Bureau; 2007.

6. Rahman MM, Haque SE, Zahan MS. Factors affecting the utilisation of postpartum care among young mothers in Bangladesh. Health Soc Care Commun. 2011;19(2):138-47. https://doi.org/10.1111/j.1365-2524.2010.00953.

7. Colbourn T, Lewycka S, Nambiar B, Anwar I, Phoya A, Mhango C. Maternal mortality in Malawi, 1977-2012. BMJ Open. 2013;3(12). https://doi.org/10.113 6/bmjopen-2013-004150.

8. National Statistical Office (NSO) [Malawi] and ICF. Malawi Demographic and Health Survey 2015-16. Zomba, Malawi, and Rockville: NSO and ICF; 2017.

9. Tiruneh GT, Worku A, Berhane Y, Betemariam W, Demissie M. Determinants of postnatal care utilization in Ethiopia: a multilevel analysis. BMC Pregnancy Childbirth. 2020;20(1):549. https://doi.org/10.1186/s12884-020-03254-7.

10. Ministry of Health [Malawi]. Health Management Information Bulletin. 2005.

11. Sakala B, Kazembe A. Factors influencing the utilisation of postnatal care at one week and six weeks among mothers at Zomba central Hospital in Malawi. Evid Based Midwifery. 2011;9(4):113-36.

12. Phiri PW, Rattanapan C, Mongkolchati A. Determinants of postnatal service utilisation among mothers in rural settings of Malawi. Health Soc Care Commun. 2015;23(5):493-501. https://doi.org/10.1111/hsc.12165.

13. Babalola S, Fatusi A. Determinants of use of maternal health services in Nigeria--looking beyond individual and household factors. BMC Pregnancy Childbirth. 2009;9(1):43. https://doi.org/10.1186/1471-2393-9-43.

14. Akunga $D$, Menya D, Kabue M. Determinants of postnatal care use in Kenya. Afr Popul Stud. 2014;28(3):1447-59.

15. Akibu M, Tsegaye W, Megersa T, Nurgi S. Prevalence and determinants of complete postnatal care service utilization in northern Shoa, Ethiopa. J Pregnancy. 2018;2018:8625437. https://doi.org/10.1155/2018/8625437.

16. Kante AM, Chung CE, Larsen AM, Exavery A, Tani K, Phillips JF. Factors associated with compliance with the recommended frequency of postnatal care services in three rural districts of Tanzania. BMC Pregnancy Childbirth. 2015;15(1):341. 
17. Berhe A, Bayray A, Berhe Y, Teklu A, Desta A, Araya T, et al. Determinants of postnatal care utilization in Tigray, northern Ethiopia: a community based cross-sectional study. PLoS One. 2019;14(8):e0221161. https://doi.org/10.13 71/journal.pone.0221161.

18. Wudineh KG, Nigusie AA, Gesese SS, Tesfu AA, Beyene FY. Postnatal care service utilization and associated factors among women who gave birth in Debretabour town, north West Ethiopia: a community- based crosssectional study. BMC Pregnancy Childbirth. 2018;18(1):508. https://doi.org/1 0.1186/s12884-018-2138- $x$

19. Workineh Y. Factors affecting utilization of postnatal care service in Jabitena district, Amhara region, Ethiopa. Sci J Public Health. 2014;23:169-76.

20. Khaki JJ, Sithole L. Factors associated with the utilization of postnatal care services among malawian women. Malawi Med J. 2019;31(1):2-11. https://doi.org/10.4314/mmj.v31i1.2.

21. Berhe H, Tilahun W, Aregay A, Bruh G, Gebremedhin HGH. Utilization and associated factors of postnatal care in Adwa town, tigray, Ethiopia-a cross sectional study a Peer Reviewed. Int J Pharm Allied Res. 2012;3(1):353-9.

22. Limenih MA, Endale ZM, Dachew BA. Postnatal care service utilization and associated factors among women who gave birth in the last 12 months prior to the study in Debre Markos town, northwestern Ethiopia: a community-based cross-sectional study. Int J Reprod Med. 2016;2016:1-7. https://doi.org/10.1155/2016/7095352.

23. Abel Ntambue ML, Françoise Malonga K, Dramaix-Wilmet M, Donnen $P$. Determinants of maternal health services utilization in urban settings of the Democratic Republic of Congo--a case study of Lubumbashi City. BMC Pregnancy Childbirth. 2012:12(1):66. https://doi.org/10.1186/1471-2393-12-66.

24. Alemayeh $\mathrm{H}$, Assefa $\mathrm{H}$, Adama Y. Prevalence and factors associated with postnatal care utilization in abi - adi town, Tigray, Ethiopia: a cross sectional study. Int J Pharm Biol Sci Fundam. 2014;8:2278-3997.

25. Tesfahun F, Worku W, Mazengiya F, Kifle M. Knowledge, perception and utilization of postnatal care of mothers in Gondar Zuria District, Ethiopia: a cross-sectional study. Matern Child Health J. 2014;18(10):2341-51. https://doi.org/10.1007/s10995-014-1474-3.

26. Angore BN, Tufa EG, Bisetegen FS. Determinants of postnatal care utilization in urban community among women in Debre Birhan town, northern Shewa, Ethiopa. J Health Popul Nutr. 2018;37(1):10. https://doi.org/10.1186/ s41043-018-0140-6.

27. Kish L. Survey sampling. Biom J. 1968;10(1):88-9.

28. StataCorp. Stata statistical software: release 12. College Station: StataCorp LP; 2011.

29. Johnston R, Jones K, Manley D. Confounding and collinearity in regression analysis: a cautionary tale and an alternative procedure, illustrated by studies of British voting behaviour. Qual Quant. 2018;52(4):1957-76. https://doi.org/10.1007/s11135-017-0584-6,

30. Bursac Z, Gauss CH, Williams DK, Hosmer DW. Purposeful selection of variables in logistic regression. Source Code Biol Med. 2008;3(1):17. https://doi.org/10.1186/1751-0473-3-17.

31. Regassa N. Antenatal and postnatal care service utilization in southern Ethiopia: a population-based study. Afr Health Sci. 2011;11(3):390-7.

32. Jat TR, Ng N, San SM. Factors affecting the use of maternal health services in Madhya Pradesh state of India: a multilevel analysis. Int J Equity Health. 2011;10(1):59. https://doi.org/10.1186/1475-9276-10-59.

33. Nankwanga A. Factors influencing utilisation of postnatal services in Mulago and Mengo hospitals Kampala, Uganda (Mini Masters Thesis). South Africa incorporated: University of the Western Cape, South Africa; 2004.

34. Dhakal S, Chapman GN, Simkhada PP, Van Teijlingen ER, Stephens J, Raja AE. Utilisation of postnatal care among rural women in Nepal. BMC Pregnancy Childbirth. 2007;7(1):19. https://doi.org/10.1186/1471-2393-7-19.

35. Khanal V, Adhikari M, Karkee R, Gavidia T. Factors associated with the utilisation of postnatal care services among the mothers of Nepal: analysis of Nepal demographic and health survey 2011. BMC Womens Health. 2014; 14(1):19. https://doi.org/10.1186/1472-6874-14-19.

36. Bonu S, Bhushan I, Rani M, Anderson I. Incidence and correlates of 'catastrophic' maternal health care expenditure in India. Health Policy Plan. 2009;24(6):445-56. https://doi.org/10.1093/heapol/czp032.

\section{Publisher's Note}

Springer Nature remains neutral with regard to jurisdictional claims in published maps and institutional affiliations.

\section{Ready to submit your research? Choose BMC and benefit from}

- fast, convenient online submission

- thorough peer review by experienced researchers in your field

- rapid publication on acceptance

- support for research data, including large and complex data types

- gold Open Access which fosters wider collaboration and increased citations

- maximum visibility for your research: over $100 \mathrm{M}$ website views per year

At BMC, research is always in progress.

Learn more biomedcentral.com/submissions 